\title{
LEEM investigations of clean surfaces driven by energetic ion beams
}

\author{
Peter Abbamonte (formerly, C. Peter Flynn) \\ abbamonte@mrl.illinois.edu \\ Department of Physics and Frederick Seitz Materials Research Laboratory \\ University of Illinois, Urbana, IL, 61801
}

Award No.: DE-FG02-08ER46549

Period: $08-16-2008$ to $10-14-2012$

Funds anticipated at end of current period: $\$ 0 \mathrm{k}$

Note: The original PI for this grant, C. Peter Flynn of the University of Illinois Physics Department, passed away in October of 2011.

\section{Program Scope}

The original purpose of this award was to use low-energy electron microscopy (LEEM) to explore the dynamics of surfaces of clean single crystal surfaces when driven by a beam of energetic ions. The goal was to understand the nanoscience of hyperthermal growth, surface erosion by sublimation and irradiation, operation of surface sinks in irradiated materials, diffusion on driven surfaces, and the creation of structural patterns. This project was based on a novel LEEM system constructed by C. P. Flynn, which provided real-time imaging of surface dynamics by scattering low energy electrons.

With the passing of Prof. Flynn in late 2011, this project was completed under a slightly different scope by constructing a low-energy, inelastic electron scattering ("EELS") instrument. Consistent with Flynn's original objectives for his LEEM system, this device (shown in Fig. 1) probes the dynamics of crystal surfaces. However the measurements are not carried out in real time, but instead are done in the frequency domain, through the energy lost from the probe electrons. The purpose of this device is to study the collective bosonic excitations in a variety of materials, including high temperature superconductors, topological insulators, carbon allotropes including (but not limited to) graphene, etc. The ultimate goal here is to identify the bosons that mediate interactions in these and other materials, with hopes of shedding light on the

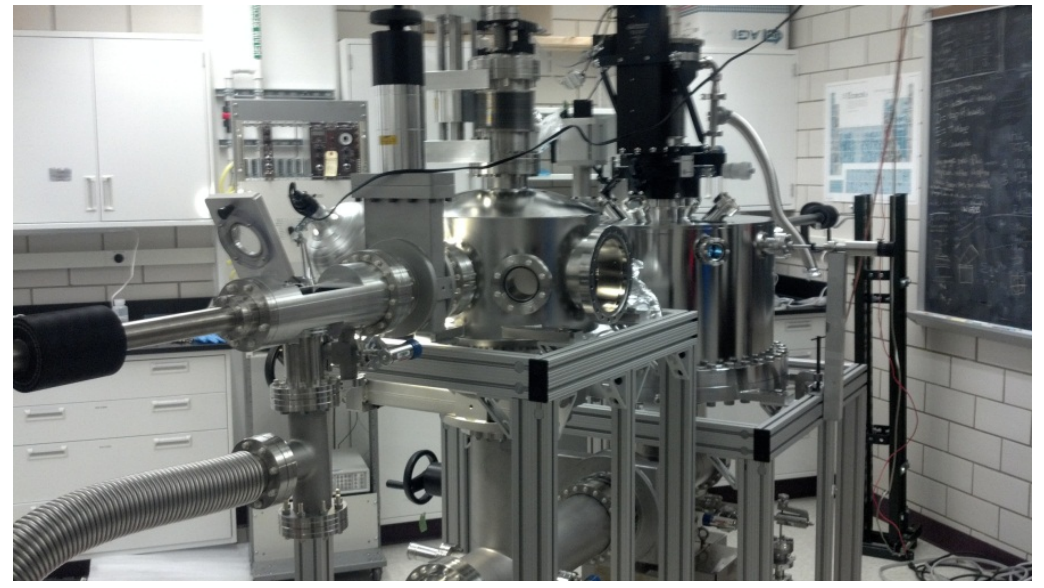

Figure 1 High-resolution electron energy loss spectrometer constructed with support from this award. This instrument operates at a beam energy of $10 \mathrm{eV}$ and exhibits an energy resolution of better than $2 \mathrm{meV}$, allowing one to detect the fundamental, low-energy collective excitations in materials. 
origin of many exotic phenomena including high temperature superconductivity.

\section{Recent Progress}

Under this award we completed the construction of a low-energy EELS system that operates with an electron kinetic energy of 7-10 eV. This instrument is fitted with a fast-entry load lock, a preparation chamber with a LEED system for monitoring the surface quality, a cryostat with a base temperature of $4 \mathrm{~K}$, and a full sample goniometer comprising both stepper motors and piezo stages. The instrument is fully automated and capable of true, reciprocal space scans, and currently exhibits an energy resolution of $2.2 \mathrm{meV}$. With this instrument now running, we hope to identify, among other things, the bosons that mediate pairing in high temperature superconductors

Using this

instrument, we have already made our first discovery. Studying freshly cleaved single crystals of $\mathrm{Bi}_{2} \mathrm{Se}_{3}$, which is a topological insulator, we have observed a surface excitation at an energy loss of 90 meV (see Fig. 2).

This excitation disperses quadratically, exhibits a critical momentum of $\mathrm{q}_{\mathrm{c}}=$ $0.11 \AA^{-1}$, and may be identified as the surface collective mode of the helical Dirac liquid, as predicted by Raghu and co-workers.[1]

To make a stronger connection between the behavior of this excitation and the known surface physics of $\mathrm{Bi}_{2} \mathrm{Se}_{3}$, we are carrying out a doping-dependent study, as a function of Se vacancy content, of this excitation. From this study we will be able to quantify the strength of interactions in the spin-polarized surface states in a manner analogous to our past work on graphene.[2]

\section{Planned activities for the next year}

We are not submitting a renewal proposal for this award, so no further activities are planned.

\section{Award-Sponsored Publications, 07-2010 to 06-2011}

1. W. Swiech, M. Ondrejcek, C. P. Flynn, LEEM investigations of ion beam effects on clean metal surfaces: quantitative studies of the driven steady state, J. Phys. Cond. Mat. 21, 314021 (2009)

2. M. Ondrejcek, W. Swiech, I. Petrov, M. Rajappan, C. Peter Flynn, Microscopy Research and Technique 72, 197 (2009) 


\section{Award-Sponsored Manuscripts in Preparation}

1. A. Kogar, S. Vig, Y. Gan, T. L. Hughes, A. Thaler, G. MacDougall, P. Abbamonte, Observation of a surface collective mode in $\mathrm{Bi}_{2} \mathrm{Se}_{3}$ with high-resolution electron energy loss spectroscopy, in preparation

Award-Sponsored Invited Talks

N/A

Personnel Supported by this Activity

W. Sweich, Senior Research Scientist, 50\%

Jacob Ruf, Academic Hourly

Da Wei, Academic Hourly

\section{References}

1. S. Raghu, S. B. Chung, X.-L. Qi, S.-C. Zhang, Collective modes of a helical liquid, Phys. Rev. Lett. 104, 116401 (2010)

2. J. P. Reed, B. Uchoa, Y. I. Joe, Y. Gan, D. Casa, E. Fradkin, P. Abbamonte, The effective finestructure constant of freestanding graphene measured in graphite, Science 330, 805 (2010) 


\section{Current and Pending Support}

Investigator: Peter M. Abbamonte

Support: $\bigotimes$ Current $\square$ Pending $\square$ Submission Planned in Near Future Project/Proposal Title: Resonant Soft X-Ray Scattering Studies of Spontaneous and Engineered

Electronic Order in Transition Metal Oxides (current activity)

Funds from this award support resonant soft x-ray scattering experiments at beam line X1B at the National Synchrotron Light Source, as described in this proposal. This program will relocate to Sector 29 at the Advanced Photon Source in 2012. See item \#3 below.

Source of Support: Department of Energy

Total Award Amount: \$1,310,684

Total Award Period Covered: 07/01/06 - 06/30/12

Location of Project: University of Illinois at Urbana-Champaign

Person-Months Per Year Committed to the Project.

Cal:

Acad:

Sumr: 1.00

Support: $\bigotimes$ Current $\square$ Pending $\square$ Submission Planned in Near Future

Project/Proposal Title: Quantum Materials at the Nanoscale (12 Investigators)

Funds from this award support hard x-ray inelastic x-ray scattering experiments at the Advanced Photon Source, high pressure hard x-ray scattering experiments in our laboratory in Urbana, and electron-beam fabrication of transition metal oxide quantum wires. None of these funds are used to support soft x-ray work.

Source of Support: Department of Energy

Total Award Amount (Investigator): \$650,528

Total Award Amount: \$5,863,590

Total Award Period Covered: 08/01/10 - 07/31/13 Location of Project: University of Illinois at Urbana-Champaign

Person-Months Per Year Committed to the Project.

Support: $\triangle$ Current $\square$ Pending $\square$ Submission Planned in Near Future Project/Proposal Title: IMR-MIP Construction of a Beamline for Soft X-Ray Photoemission and Scattering

This award, which is not renewable, supports construction of an endstation for the new IEX beam line in Sector 29 at the APS. This facility will be the destination of the activity described in this proposal and item \#1 in this list.

Source of Support: National Science Foundation

Total Award Amount: \$807,589

Total Award Period Covered: 10/01/07 - 09/30/11

Location of Project: University of Illinois at Urbana-Champaign

Person-Months Per Year Committed to the Project.

Support: $\bigotimes$ Current $\square$ Pending $\square$ Submission Planned in Near Future Project/Proposal Title: Center for Emergent Superconductivity (10 Illinois Investigators)

This activity supports polarized x-ray scattering experiments to search for loop currents in copperoxides. This project is part of the EFRC run by Brookhaven National Lab., Argonne National Lab., and the University of Illinois. These experiments are a collaboration with Seamus Davis at Cornell / BNL and are carried out at CHESS. None of these funds are used to support soft x-ray work.

Source of Support: Brookhaven National Laboratory (DOE/EFRC)

Total Award Amount (Investigator): \$432,755 Total Award Period Covered: 08/14/09 - 08/13/14

Total Award Amount (Illinois): \$6,216,000

Location of Project: University of Illinois at Urbana-Champaign

Person-Months Per Year Committed to the Project.

Cal:

Acad:

Sumr: 0.50 